\title{
Ceramic Membrane Immobilized Salen Catalysts and Their Use in Asymmetric Catalytic Reactions
}

\author{
Liu Min, Zhao Zhi-Ping*, Li Jian-Hui \\ School of Chemical Engineering and the Environment \\ Beijing Institute of Technology \\ Beijing, PR China \\ e-mail: zhaozp@bit.edu.cn.
}

\begin{abstract}
Three kinds of salen catalysts, monochloromethyl-salen-Mn, tetra-tert-butyl-salen-Mn and mono-chloromethyl-slaen-Co, were synthesized and then immobilized onto ceramic membranes by two steps. First, 3aminopropyltriethoxysilane (APTES) was grafted on ceramic membranes in order to introduce amino groups onto the membranes. Second, catalysts were immobilized onto the membranes through chemical bonding of active groups of the catalysts and amino groups on the membranes. And three membrane reactors corresponding to the three catalysts were constructed. The catalytic performances of the reactors were investigated by catalyzing the epoxidation of indene and hydrolytic kinetic resolution (HKR) of racemic epichlorohydrin. The conversion rates (Conv.) and ee values of mono-chloromethyl-salen-Mn and tetra-tert-butyl-salenMn membrane reactors were $31.1 \% / 28.9 \%$ (Conv.) and $76.1 \% / 74.3 \%$ (ee) respectively in catalyzing epoxidation of indene. The Conv. and ee values of mono-chloromethylsalen-Co membrane reactor were $39.4 \%$ and $24.5 \%$ respectively in catalyzing $\mathrm{HKR}$ reaction of epichlorohydrin. The salen membrane reactors showed comparable or even higher catalytic efficiency with the corresponding homogeneous catalysts. And all the reactors can reuse the catalysts directly without separation stage.
\end{abstract}

Keywords-salen-Mn catalyst; immobilization; membrane reactor; epoxidation; Hydrolytic kinetic resolution

\section{INTRODUCTION}

Asymmetric catalytic reactions can provide useful building blocks for many kinds of natural products, optical materials and optically active drugs ${ }^{[1]}$. Chiral salen catalysts are one kind of excellent catalyst in asymmetric reactions. They can catalyze various kinds of reactions, such as oxidation kinetic resolution of racemic compounds ${ }^{[2,3]}$, asymmetric cyclopropanation of olefin ${ }^{[4]}$, strecker reaction ${ }^{[5,6]}$ asymmetric epoxidation of thioether ${ }^{[7,8]}$ and styrene ${ }^{[9,10]}$, and show outstanding catalytic performances. But it is difficult to separate this kind of catalyst from the reaction mixture, so lots of reports focused on the immobilization of the catalysts to resolve this problem ${ }^{[11-15]}$. Catalyst immobilization can not only simplify the separation operation of the catalyst, but also improve the atom utilization of the catalyst, which meets the requirement of green chemistry. But the separation of the immobilized catalysts, especially for ultrafine heterogeneous catalysts, is still a challenge in industrial application ${ }^{[16-18]}$. Besides, the heterogeneous catalysts cannot be dispersed well in the reaction systems, which will have a negative influence on the mass transfer of the reaction. If the catalysts were immobilized onto membranes and the membranes were assembled as a reactor, the described problems could be resolved effectively ${ }^{[19,20]}$.

In our previous work ${ }^{[21]}$, mono-chloromethyl-salen-Mn catalyst (Fig. 1) was immobilized onto amino-modified ceramic membrane and membrane reactor (Fig. 2) was constructed. The reactor showed comparable catalytic properties in catalyzing epoxidation of styrene. And the membrane catalyst can be reused directly only by washing the membrane with fresh solvent without being unloaded from the reactor.

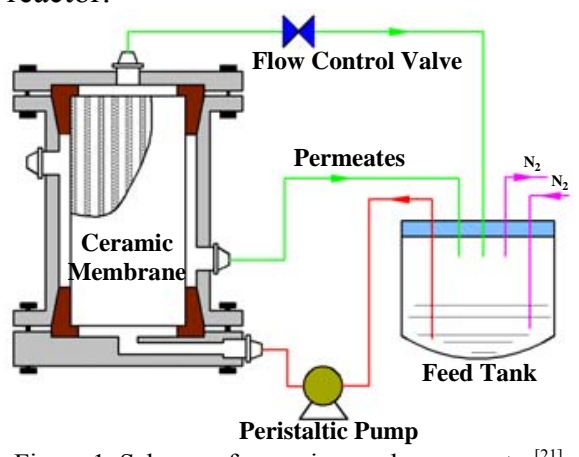

Figure 1. Scheme of ceramic membrane reactor ${ }^{[21]}$

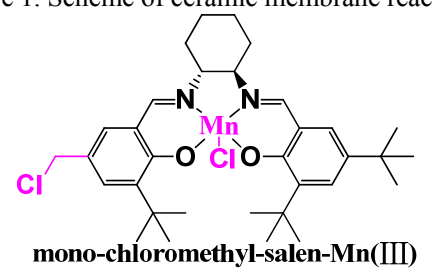

Figure 2. Structure of mono-chloromehyl-salen-Mn ${ }^{[21]}$

In this work, the mono-chloromethyl-salen-Mn membrane was used to catalyzing asymmetric epoxidation of indene and tetra-tert-butyl-salen-Mn (Fig. 3, a) membrane reactor was constructed in order to compare the catalytic properties of the two reactors. Monochloromethyl-salen-Co (Fig. 3, b) ceramic membrane reactor was also constructed by the similar method and 
used in catalyzing hydrolytic kinetic resolution (KHR) of epichlorohydrin.

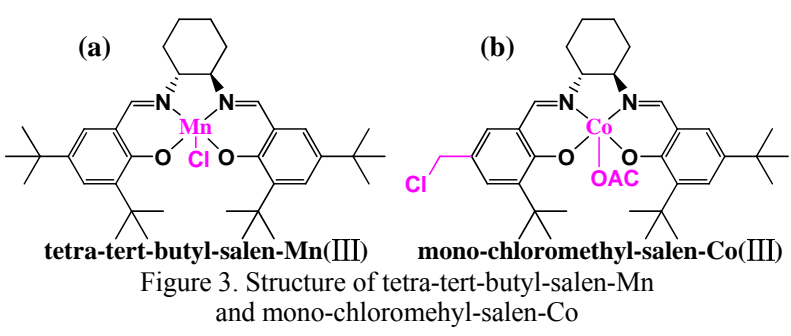

\section{EXPERIMENTAL SECTION}

\section{A. Materials}

Ceramic membranes $\left(\mathrm{CMs}, \mathrm{ZrO}_{2}\right.$ top layer, $\mathrm{Al}_{2} \mathrm{O}_{3}$ supporting layer; external diameter $30 \mathrm{~mm}$, pore size 70 nm, 19 channels) were purchased from Hefei Shijie Membrane Engineering Co., Ltd. Unless otherwise noted, all chemicals were analytical reagent (AR) and used as received. 2-tert-butylphenol, 3,5-di-tert-butyl-2hydroxybenzaldehyde, paraformaldehyde, manganese (III) acetate dehydrate, Cobalt(II) acetate, hexamethylenetetramine, tin (II) chloride, 3chloroperoxybenzoic acid (m-CPBA), 4-methylmorpholine N-oxide (NMO), lithium chloride and 3aminopropyltriethoxysilane (APTES) were purchased from Acros Chemical Company; (1R,2R)-(-)-1,2diaminocyclohexane from Shandong Boyuan Pharmaceutical Co., Ltd; Concentrated sulfuric acid, concentrated hydrochloric acid and glacial acetic acid from Tianjin Bodi Chemical Holding Co., Ltd.; $\mathrm{MgSO}_{4}$ from Beijing East Longshun Chemistry Syntheses Technique Center; Methanol, ethanol, petroleum ether , toluene, anhydrous potassium carbonate, dichloromethane, sodium chloride and sodium bicarbonate from Beijing Chemical Plant. Toluene and dichloromethane were dehydrated by calcium hydride before used. The salen catalysts were synthesized according to the method in reference [21].

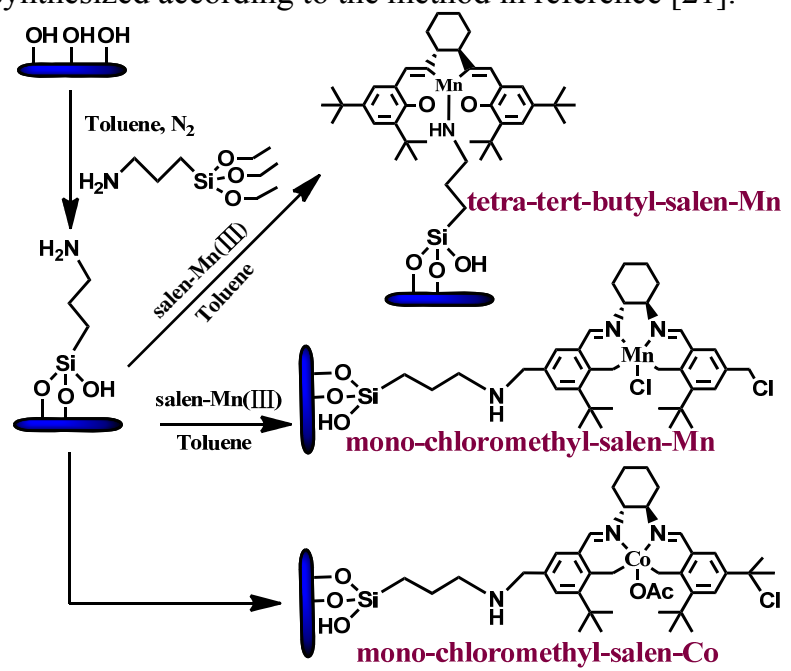

Figure 4. Routes of grafting APTES and

immobilizing salen catalyst onto ceramic membrane

\section{B. Modification of Ceramic Membranes by grafting} APTES

The grafting route is showed in Fig. 4. First, ceramic membrane activated by diluted hydrochloric acid solution was packed as a reactor showed in Fig. 1. Then dichloromethane and APTES were added and cycled at room temperature for 48 hours under the protection of $\mathrm{N}_{2}$. Third, the reaction system was washed by fresh dichloromethane thoroughly, and the modified membranes were named as APTES-g-CMs.

\section{Immobilization of Chiral Salen Catalysts}

The immobilization routes of salen catalyst are showed in Fig. 4. Salen catalyst $(1 \mathrm{~g})$ and toluene $(60 \mathrm{ml})$ were added into the feed tank, the reaction solution was cycled at $80^{\circ} \mathrm{C}$ for 20 hours. When finished, the system was first washed by toluene until the solution was transparent, then washed by water until the solution was neutral. The immobilization degrees of the salen catalysts were tested by ultraviolet spectrophotometric method (UNICO, UV4802) with ethanol as the reference solution.

\section{Characterization Methods}

X-ray photoelectron spectroscopy (XPS) analysis was performed on a PHI QUANTEAR- II equipped with a monochromatic $\mathrm{Al} \mathrm{K} \alpha$ radiation $(1486.6 \mathrm{eV})$ at an operating power of $25 \mathrm{~W}$. The measurements have been performed at a takeoff angle (TOA) fixed at $45^{\circ}$ (measured with respect to the sample surface). For calibration, the carbon $\mathrm{C} 1 \mathrm{~s}$ peak was used $(284.8 \mathrm{eV})$.

\section{E. Epoxidation of Indene}

The reaction formula of epoxidation of indene is showed in Fig. 5. The reactor was precooled to $0^{\circ} \mathrm{C}$, then indene (4 mmol), NMO (20 mmol, as axial ligand), toluene $(0.1 \mathrm{~g}$, as internal standard substance) and dichloromethane $(55 \mathrm{ml})$ were added into the feed tank. The mixture was cycled for 15 minutes. Then m-CPBA $(1 \mathrm{mmol})$ was added by 4 times in one hour. The reaction was maintained at $0{ }^{\circ} \mathrm{C}$ for 24 hours, and was detected periodically by $\mathrm{GC}$ (TECHCOMP 7890II), using a RESTEK Rt-bDEXse instrument equipped with a $30 \mathrm{~m} \times 0.25 \mathrm{~mm} \times 0.25 \mu \mathrm{m}$ film thickness silica capillary columns.

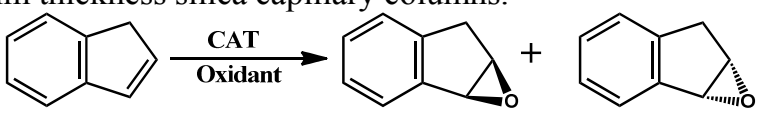

Figure 5. Scheme of epoxidation of indene

\section{F. HKR Reaction of Epichlorohydrin}

Epichlorohydrin $(1 \mathrm{ml})$, methyl isobutyl ketone $(0.1 \mathrm{~g}$, as internal standard substance), water $(0.15 \mathrm{ml})$ and $\mathrm{THF}$ $(50 \mathrm{ml})$ were added into the feed tank. The reaction mixture was cycled for 70 hours at $20{ }^{\circ} \mathrm{C}$ and was detected periodically by GC (TECHCOMP 7890II), using a GAMMA DEXTM225, $30 \mathrm{~m} \times 0.25 \mathrm{~mm} \times 0.25 \mu \mathrm{m}$ film thickness silica capillary columns. The reaction formula was showed in Fig. 6.

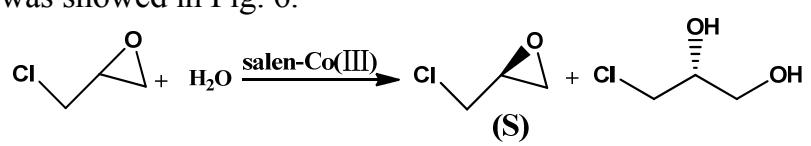

Figure 6. Scheme of HKR of epichlorohydrin

\section{RESULTS AND DISCUSSION}

\section{A. Catalytic properties of mono-chloromethyl-salen-Mn}

The catalytic properties of homogeneous monochloromethyl-salen-Mn catalyst and the corresponding 
membrane reactor were investigated by catalyzing the asymmetric epoxidation of indene. The results are showed in TABLE I and TABLE II respectively. The catalytic activity and enantioselectivity of the mono-chloromethylsalen-Mn catalyst decreased after immobilized onto the membrane, which may attributed to the low immobilization degree of the catalyst. And the space structure of the catalyst was influenced by the membrane support, which may make a negative effect on the enantioselectivity of the catalyst. However, the catalytic efficiency of the membrane reactor was comparable to the homogeneous catalyst. And the membrane catalyst can be reused only by washing the membrane with fresh solvent after each reaction cycle without unloading the membrane. The stability of the membrane catalyst was test by reusing the reactor for three times, and the results (TABLE III) showed no obvious change on conbersion rate (Conv.) and ee values. So the total catalytic efficiency of the immobilized salen-Mn catalyst is much higher than the corresponding homogeneous catalyst since the membrane reactor can reuse the catalyst for more than three times.

TABLE I. EPOXIDATION OF INDENE CATALYZED BY HOMOGENEOUS MONO-CHLOROMETHYL-SALEN-MN

\begin{tabular}{cccc}
\hline Time $(\mathrm{h})$ & Conv. $(\%)$ & ee $(\%)$ & TOF $\left(10^{-2} \mathrm{~S}^{-1}\right)$ \\
\hline 2 & 90.3 & 93.3 & 1.2122 \\
3 & 93.3 & 92.9 & 0.8332 \\
4 & 95.1 & 93.6 & 0.6393 \\
5 & 95.3 & 93.2 & 0.5114 \\
9 & 96.1 & 93.4 & 0.2868 \\
\hline
\end{tabular}

Mol ratio of indene/catalyst $=1 \%$ TOF $=$ mol of product $/$ catalyst $/$ time

TABLE II. EPOXIDATION OF INDENE CATALYZED BY MONOCHLOROMETHYL-SALEN-MN MEMBRANE REACTOR

\begin{tabular}{cccc}
\hline Time $(\mathrm{h})$ & Conv. $(\%)$ & ee $(\%)$ & TOF $\left(10^{-2} \mathrm{~S}^{-1}\right)$ \\
\hline 2 & 12.2 & 75.8 & 1.1549 \\
8.5 & 18.4 & 75.7 & 0.4094 \\
16.5 & 24.9 & 75.4 & 0.2850 \\
24 & 30.0 & 76.1 & 0.2370 \\
26.5 & 31.1 & 76.1 & 0.2225 \\
\hline \multicolumn{4}{c}{ Mol ratio of indene/catalyst $=0.129 \%$}
\end{tabular}

TABLE III. STABILITY TEST OF MONO-CHLOROMETHYLSALEN-MN MEMBRANE REACTOR

\begin{tabular}{ccccc}
\hline Run & Time $(\mathrm{h})$ & Conv. $(\%)$ & ee $(\%)$ & TOF $\left(10^{-2} \mathrm{~S}^{-1}\right)$ \\
\hline 1 & 26.5 & 31.1 & 76.1 & 0.2225 \\
2 & 26.5 & 30.7 & 76.3 & 0.2111 \\
3 & 26.5 & 30.1 & 75.7 & 0.2067 \\
\hline
\end{tabular}

B. Catalytic properties of tetra-tert-butyl-salen-Mn

In order to investigate the influence of immobilization method on the catalytic performance of the membrane reactor, tetra-tert-butyl-salen-Mn catalyst was immobilized onto amino-modified ceramic membrane through the chemical bonding of metal center of catalyst and amino group of membrane. Both of the homogeneous catalyst and membrane reactor were used in catalyzing the epoxidation of indene. The results are showed in TABLE IV and TABLE $V$ respectively. The catalytic efficiency of the membrane reactor was comparable to the homogeneous catalyst. In compared with mono-chloromethyl-salen-Mn reactor, the catalytic activity of tetra-tert-butyl-salen-Mn reactor was slightly low, which may attribute the lower immobilization degree. And the attachment of catalytic metal center to the support may influence the space structure of the catalyst, so the ee value was lower too. The results suggest that, the way of immobilizing catalyst through chemical bonding of the active groups of the catalyst ligand with the membrane is better than through coordinate bond of the metal center of the catalyst to the membrane.

TABLE IV. EPOXIDATION OF INDENE CATALYZED BY HOMOGENEOUS TETRA-TERT-BUTYL-SALEN-MN

\begin{tabular}{cccc}
\hline Time $(\mathrm{h})$ & Conv. $(\%)$ & $\mathrm{ee}(\%)$ & $\mathrm{TOF}\left(10^{-2} \mathrm{~S}^{-1}\right)$ \\
\hline 2 & 94.8 & 93.1 & 1.2712 \\
3 & 95.9 & 93.2 & 0.8578 \\
4 & 96.9 & 93.8 & 0.6521 \\
5 & 97.6 & 93.9 & 0.5257 \\
9 & 99.6 & 93.3 & 0.2971 \\
\hline
\end{tabular}

Mol ratio of indene/catalyst $=1 \%$

TABLE V. EPOXIDATION OF INDENE CATALYZED BY TETRA-TERT-BUTYL-SALEN-MN MEMBRANE REACTOR

\begin{tabular}{cccc}
\hline Time (h) & Conv. (\%) & ee (\%) & TOF $\left(10^{-2} \mathrm{~S}^{-1}\right)$ \\
\hline 2 & 12.3 & 74.8 & 1.2340 \\
8.5 & 17.9 & 74.7 & 0.4223 \\
16.5 & 23.4 & 74.6 & 0.2842 \\
24 & 27.3 & 74.6 & 0.2280 \\
26.5 & 28.9 & 74.3 & 0.2182 \\
\hline \multicolumn{4}{c}{}
\end{tabular}

C. Catalytic properties of mono-chloromethyl-salen-Co

In order to investigate the suitability of membrane reactor for other kind of salen catalyst, monochloromethyl-salen-Co was immobilized on to ceramic membrane. The homogeneous catalyst and the correspondent membrane reactor were applied in catalyzing the HKR reaction of epichlorohydrin. The results are showed in TABLE VI and TABLE VII respectively. The catalytic activity of the salen-Co catalyst decreased after immobilizing onto the ceramic membrane. But the catalytic efficiency of the membrane reactor was higher than the homogeneous counterpart. The further research should focus on improving the immobilization degree of the catalyst by optimizing the grafting and immobilizing reaction conditions.

TABLE VI. HKR REACTION OF EPICHLOROHYDRIN CATALYZED BY HOMOGENEOUS MONO-CHLOROMETHYLSALEN-CO

\begin{tabular}{cccc}
\hline Time $(\mathrm{h})$ & Conv.(\%) & ee $(\%)$ & TOF $\left(10^{-2} \mathrm{~S}^{-1}\right)$ \\
\hline 2 & 27.2 & 25.5 & 0.3585 \\
10 & 74.2 & 69.7 & 0.1957 \\
20 & 86.1 & 80.9 & 0.1136 \\
30 & 97.4 & 91.6 & 0.0857 \\
40 & 99.7 & 96.0 & 0.0658 \\
\hline
\end{tabular}

Mol ratio of epichlorohydrin/catalyst $=0.6 \%$ 
TABLE VII. HKR REACTION OF EPICHLOROHYDRIN CATALYZED BY MONO-CHLOROMETHYL-SALEN-CO MEMBRANE REACTOR

\begin{tabular}{cccc}
\hline Time $(\mathrm{h})$ & Conv. $(\%)$ & ee $(\%)$ & TOF $\left(10^{-2} \mathrm{~S}^{-1}\right)$ \\
\hline 1 & 3.4 & 3.2 & 0.6734 \\
5 & 7.2 & 6.8 & 0.2862 \\
10 & 10.8 & 10.3 & 0.2168 \\
20 & 15.6 & 14.8 & 0.1557 \\
30 & 19.5 & 18.5 & 0.1298 \\
40 & 22.9 & 21.7 & 0.1142 \\
50 & 24.1 & 22.9 & 0.0964 \\
60 & 24.8 & 23.6 & 0.0828 \\
70 & 25.7 & 24.5 & 0.0737 \\
\hline
\end{tabular}

Mol ratio of epichlorohydrin/catalyst $=0.132 \%$

\section{CONCLUSIONS}

Mono-chloromethyl-salen-Mn, tetra-tert-butyl-salen$\mathrm{Mn}$ and mono-chloromethyl-slaen-Co catalytic membrane reactors were constructed by immobilizing the catalysts onto amino-grafted ceramic membranes. The catalytic performances of the two kinds of salen-Mn membrane reactors were investigated by catalyzing the epoxidation of indene, and the reactors showed comparable catalytic efficiency with the corresponding homogeneous catalysts. The salen-Co reactor was used in HKR of racemic epichlorohydrin, and showed much higher catalytic efficiency in contrast to the homogeneous counterpart. All of the reactors can reuse the catalysts directly without separation operations. And the comparison of the results of asymmetric epoxidation of indene catalyzed by the tetratert-butyl-salen-Mn( III ) membrane reactor and monochlorolymethyl-salen-Mn(III) membrane reactor suggested that, the way of immobilizing catalyst through the groups of catalyst ligand to the membrane is better than through coordinate bond of metal center of the catalyst to the membrane.

\section{ACKNOWLEDGMENT}

The authors would like to thank the supporting of the National Nature Science Foundation of China (No.20676015) and (No.21276024).

\section{REFERENCES}

[1] White D E, Jacobsen E N. "New oligomeric catalyst for the hydrolytic kinetic resolution of terminal epoxides under solventfree conditions"'J]. Tetrahedron: Asymmetr, Vol. 14, pp. 36333638, 2003.

[2] Tokunaga M. "Asymmetric catalysis with water: efficient kinetic resolution of terminal epoxides by means of catalytic hydrolysis"[J]. Science, Vol. 277, pp. 936-938, 1997.

[3] Nishibayashi A Y Y, Onodera G, Uemura S. "Oxidative kinetic resolution of racemic alcohols catalyzed by chiral ferrocenyloxazolinylphosphine-ruthenium complexes" $[\mathrm{J}]$. J Org Chem, Vol. 68, pp. 5875-5880, 2003.

[4] Niimi T, Uchida T, Irie R, et al. "Co(II)-salen-catalyzed highly cisand enantioselective cyclopropanation" $[\mathrm{J}]$. Tetrahedron Lett, Vol. 41, pp. 3647-3651, 2000.

[5] Khan N H, Saravanan S, Rukhsana I, et al. "Mn(III) salen complexes-catalyzed enantioselective addition of trimethyl silylcyanide to N-benzylimines in the presence of 4-phenyl pyridine-N-oxide as an additive"'[J]. Tetrahedron: Asymmetr, Vol. 21, pp. 2076-2080, 2010.

[6] Khan N H, Saravanan S, Rukhsana I, et al. "Asymmetric addition of trimethylsilylcyanide to N-benzylimines catalyzed by recyclable chiral dimeric V(V) salen complex"[J]. J Organomet Chem, Vol. 695, pp. 1133-1137, 2010.

[7] Palucki M, Hanson P, Jacobsen E N. Asymmetric oxidation of sulfides with $\mathrm{H}_{2} \mathrm{O}_{2}$ catalyzed by (salen)Mn(III) complexes[J]. Tetrahedron Lett, Vol. 33, pp. 7111-7114, 1992.

[8] Nakajima K, Sasaki C, Kojima M, et al. "Preparation and characterization of optically active quadridentate schiff basetitanium( IV ) compleses and the catalytic properties of these compleses on asymmetric oxidation of methyl phenyl sulfide with organic hydroperoxides"[J]. Bull Chem Soc Jpn, Vol. 64, pp. 13181324, 1991.

[9] Kureshy R I, Khan N H, Hasan S , et al. Enantioselective epoxidation of non-functionalised alkenes catalysed by dimeric homochiral Mn(III) Salen complex using oxone as oxidant[J]. J Mol Catal A: Chem, Vol. 203, pp. 69-73, 2003.

[10] Kureshy R I, Khan N H, Abdi S H R, et al. "Catalytic asymmetric epoxidation of non-functionalised alkenes using polymeric $\mathrm{Mn}(\mathrm{III})$ salen as catalysts and $\mathrm{NaOCl}$ as oxidant"'[J]. J Mol Catal A: Chem, Vol. 218, pp. 141-146, 2004

[11] Biernacka I K, Pereira C, Carvalho A P, et al. "Epoxidation of olefins catalyzed by manganese(III) salen complexes grafted to porous heterostructured clays"[J]. Applied Clay Science, Vol. 53, pp. 195-203, 2011.

[12] Biernacka I K, Silva A R, Carvalho A P, et al. "Anchoring of chiral manganese(iii) salen complex onto organo clay and porous clay heterostructure and catalytic activity in alkene epoxidation"[J]. Catal Lett, Vol. 134, pp. 63-71, 2009.

[13] Zhang H D, Xiang S, Xiao J L, et al. "Heterogeneous enantioselective epoxidation catalyzed by $\mathrm{Mn}$ (salen) complexes grafted onto mesoporous materials by phenoxy group" [J]. J Mol Catal A: Chem, Vol. 238, pp. 175-184, 2005.

[14] Lou L L, Jiang S, Yu K, et al. "Mesoporous silicas functionalized with aminopropyl via co-condensation: effective supports for chiral $\mathrm{Mn}(\mathrm{III})$ salen complex"[J]. Micropor Mesopor Mat, Vol. 142, pp. 214-220, 2011.

[15] Ayala V, Corma A, Iglesias M, et al. "Mesoporous MCM41heterogenised (salen) $\mathrm{Mn}$ and $\mathrm{Cu}$ complexes as effective catalysts for oxidation of sulfides to sulfoxides"[J]. J Mol Catal A: Chem, Vol. 221, pp. 201-208, 2004.

[16] Zhong Z X, Xing W H, Jin W Q, et al. "Adhesion of nanosized nickel catalysts in the nanocatalysis/UF system”[J]. AIChE J, Vol. 53, pp. 1204-1210, 2007.

[17] Lu C J, Chen R Z, Xing W h, et al. "A submerged membrane reactor for continuous phenol hydroxylation over TS-1"[J]. AIChE J, Vol. 54, pp. 1842-1849, 2008.

[18] Chen R Z, Jiang Y G, Xing W H, et al. "Fabrication and catalytic properties of palladium nanoparticles deposited on a silanized asymmetric ceramic support'[J]. Ind Eng Chem Res, Vol. 50, pp. 4405-4411, 2011.

[19] Dittmeyer R, Svajda K, Reif M. A review of catalytic membrane layers for gas/liquid reactions[J]. Top Catal, Vol. 29, pp. 3-27, 2004.

[20] Miachon S, Dalmon J A. "Catalysis in membrane reactors: what about the catalyst?’[J]. Top Catal, Vol. 29, pp. 59-65, 2004.

[21] Liu, M.; Zhao, Z. P.; Chen, K. C.; Liu, W. F. "New chiral catalytic membrane reactor created by immobilizing salen-Mn(III) onto APTES modified ceramic membrane and its performances in epoxidation of styrene" [J]. Catal. Commun, Vol. 64, pp. 70-74, 2015 . 